Cahiers de philosophie de l'université de

\title{
L'idée de surhomme
}

\section{Vladimir Soloviev}

Traducteur : Antoine Muller

\section{CpenEdition}

\section{Journals}

Édition électronique

URL : https://journals.openedition.org/cpuc/1042

DOI : $10.4000 /$ cpuc. 1042

ISSN : 2677-6529

Éditeur

Presses universitaires de Caen

Édition imprimée

Date de publication : 31 octobre 2011

Pagination : 195-204

ISBN : 978-2-84133-385-1

ISSN : 1282-6545

\section{Référence électronique}

Vladimir Soloviev, "L'idée de surhomme », Cahiers de philosophie de l'université de Caen [En ligne], 48 |

2011, mis en ligne le 01 septembre 2020, consulté le 03 février 2023. URL : http://

journals.openedition.org/cpuc/1042 ; DOI : https://doi.org/10.4000/cpuc.1042

\section{(c) (7) 8}

Creative Commons - Attribution - Pas d'Utilisation Commerciale 4.0 International - CC BY-NC 4.0

https://creativecommons.org/licenses/by-nc/4.0/ 


\section{L'idée de surhomme ${ }^{1}$}

D

ANS LA DERNIÈRE LIVRAISON de la revue philosophique moscovite Questions de philosophie et de psychologie (janvier-février 1899), analysant une traduction récente de Nietzsche, V. P. Preobrajenski, bon connaisseur et amateur de cet écrivain, remarque, entre autres choses, qu' «il semble que Nietzsche devienne, non sans quelque malheur pour lui, un écrivain à la mode en Russie; du moins est-il l'objet d'une demande manifeste» («Revue des livres», p. 48) ${ }^{2}$.

Le «malheur» d'une telle mode n'est, pourtant, que le reflet extérieur nécessaire, de ce fait intérieur qu'une certaine idée s'est réellement mise à vivre dans la conscience de la société: car avant de devenir l'objet d'une demande de marché, elle a, sans aucun doute, apporté une réponse à quelque interpellation spirituelle des personnes qui pensent.

Il y a cinquante ou soixante ans de cela, la mode était à Hegel - également non sans «quelque malheur» pour Hegel lui-même ${ }^{3}$. Pourtant, s'il s'avérait que la culture russe, hormis les fleurs enchanteresses de notre poésie, donnât encore des fruits mûrs de véritable entendement et ordonnancement de la vie, alors il conviendrait bien entendu de reconnaître comme le premier germe encore obscur de ces fruits l'hégélianisme russe des années 30-40.

Il en va de même des engouements intellectuels qui ont remplacé l'hégélianisme, et ont causé « quelque malheur» à Darwin, Comte et beaucoup d'autres. Je pense qu'il faut considérer tout cela comme des degrés transitoires, risibles pour ce qui est de leur expression extérieure, mais

1. «Идея сверхчеловека», article de Vladimir Soloviev paru pour la première fois dans la revue Мир исскуства [Le monde de l'art], 9, 1899, p. 87-91.

2. Recension de F. Nietzsche, Ainsi parlait Zarathoustra: neuf extraits, Saint-Pétersbourg, 1899 (dans la traduction de S. P. Nani); Вопросы философии и психологии [Questions de philosophie et de psychologie], $\mathrm{n}^{\circ} 46,1899$.

3. S'il est un philosophe occidental qui fit l'objet d'une réception nourrie, multiforme et controversée en Russie, c'est bien Hegel. Voir l'étude de G. Planty-Bonjour (Hegel et la pensée philosophique en Russie, 1830-1917, La Haye, M. Nijhoff, 1974), qui cite (p. 9 sq.) tout en les nuançant les témoignages critiques fournis par I. V. Kireïevski et N. F. Pavlov sur leurs contemporains, hégéliens en herbe. 
inévitables dans leur essence - comme des "passions de jeunesse", sans lesquelles ne peut advenir la véritable maturité.

Je ne regrette pas du tout que les paléosaures et les mastodontes aient été un temps l'objet le plus élevé de mon amour ${ }^{4}$. Quoique «le sentiment d'humanité pour le menu bétail», selon l'expression d'un héros de Dostoïevski ${ }^{5}$, me contraigne jusqu'à présent à ressentir quelques remords pour ces sangsues, que j'ai taillées en pièces au rasoir, en procédant à une «coupe transversale» - et ce d'autant plus que le crime fut inutile, mes exercices histologiques s'étant révélés plus néfastes pour le microscope de l'État qu'édifiants pour moi -, tout en me repentant de la vaine mise à mort de ces primaires parents, je me souviens avec reconnaissance de la passion que j'ai éprouvée. Je sais qu'elle fut utile pour moi, je pense que passer par le culte des sciences naturelles après les abstractions hégéliennes fut nécessaire et utile pour toutes les jeunes générations de la société russe.

Passant des souvenirs à ce que nous avons devant les yeux, nous noterons une différence entre les engouements intellectuels d'antan et ceux d'aujourd'hui dans la société russe. Auparavant, de telles passions se suivaient peut-être assez rapidement les unes les autres, mais à chaque moment donné, l'une d'elles régnait sans partage (quoique, bien entendu, avec toutes sortes de nuances diverses). La croissance intérieure de notre société se présentait comme une sorte de procession triomphale droit en avant, et qui ne désirait pas passer pour "arriéré» et s'exposer au mépris général devait en même temps que toutes les "personnes avancées» atteindre la même étape intellectuelle. Il y a longtemps déjà que le mouvement de notre culture a perdu ce caractère rectiligne et, si l'on peut s'exprimer ainsi, d'étapes obligatoires, premièrement parce les gens peu instruits sont beaucoup plus nombreux, et qu'il n'est pas si simple et aisé de les rassembler, et deuxièmement parce que ces gens s'avèrent sinon plus mûrs, en tout cas, moins naïfs, et en conséquence moins aptes à la "pensée unique» du troupeau. C'est pourquoi on voit de

4. Soloviev adolescent suivit les cours des facultés de sciences naturelles, puis d'histoire et philologie, avant de se retourner contre le matérialisme et le positivisme en 1875 dans son livre Crise de la philosophie occidentale.

5. Il s'agit du capitaine Lebiadkine, dans sa lettre à Lisa Touchine où il était déjà question de microscope (voir F. Dostoïevski, Les démons, Paris, Gallimard, 1955 [trad. par B. de Schloezer et S. Luneau], première partie, IV, II, p. 139). La médiation romanesque confère à la formule, citée littéralement, une tournure humoristique: «Vous êtes une déesse de l'antiquité, et moi je ne suis rien, mais j'ai le pressentiment de l'infini. [...] Le soleil peut-il se fâcher contre un infusoire si celui-ci lui adresse un poème au fond d'une goutte d'eau, où le microscope en découvre tant? Même le club de protection des grands animaux qui s'est fondé à Pétersbourg, dans la haute société, tout en éprouvant à juste titre de la compassion pour un chien ou un cheval, méprise le doux infusoire et n'y fait aucune allusion, parce qu'il est trop infime $[\ldots] »$. 
toute part des individus et des groupes fractionnés, isolés, faire route à part, sans se joindre à un mouvement plus étendu et général. Les hommes, en particulier ceux qui sont sensibles aux exigences communes de la minute présente de l'histoire, sont dominés non par une seule, mais au moins par trois idées à l'ordre du jour, ou, si l'on veut, à la mode - le matérialisme économique, le moralisme abstrait et le démonisme du "surhomme». De ces trois idées, liées à trois grands noms (Karl Marx, Léon Tolstoï, Friedrich Nietzsche), la première est tournée vers l'actuel et son urgence, la deuxième embrasse en partie les lendemains, la troisième est liée à ce qui adviendra après-demain et ensuite. Je la considère comme la plus intéressante des trois.

Chaque idée en elle-même n'est en effet qu'une lucarne intellectuelle. Dans la lucarne du matérialisme économique nous ne voyons que la cour de derrière, ou comme disent les Français, la basse-cour ${ }^{6}$ de l'histoire et de l'époque contemporaine; la fenêtre du moralisme abstrait donne sur une cour propre, et même trop propre, jusqu'à en être complètement vide, une cour de l'impassibilité, du dépouillement, de la non-résistance, de la nonintervention et autres « in-» et «non-», tandis que la fenêtre nietzschéenne du "surhomme» ouvre directement un espace immense pour toutes sortes de chemins de vie, et s'il advient que quelqu'un en s'élançant sans regarder en arrière dans cet espace tombe dans un trou, ou s'embourbe dans un marais ou dans un précipice pittoresque, grandiose, mais sans issue, ces directions ne constitueront une nécessité inconditionnelle pour personne, chacun est libre de choisir le chemin sûr et magnifique, au bout duquel luisent déjà au loin dans le brouillard des sommets aériens illuminés par un soleil éternel7.

Ce n'est pas d'un point de vue philosophique ou historique que je veux maintenant analyser le nietzschéisme, je veux seulement lui appliquer la première condition d'une vraie critique: montrer le principe fondamental du phénomène intellectuel analysé - autant que faire se peut - de son bon côté.

I. Je pense qu'il va de soi que toute erreur - du moins toute erreur qui vaut la peine qu'on parle d'elle - contient une vérité indubitable et n'est que l'altération plus ou moins profonde de cette vérité; c'est elle qui lui sert de support, c'est d'elle qu'elle tient son attrait et son danger, c'est par son biais seulement qu'elle peut être comme il convient comprise, évaluée et définitivement réfutée.

6. Le texte russe donne le terme français entre parenthèses.

7. On rapprochera ces lignes des derniers vers d'une poésie de Soloviev parue en 1884 : «J'irai vers le sommet où sous de nouveaux astres, / Flamboyant dans les feux d'une lumière triomphante, / Se dresse mystérieux le temple qui m'attend", Anthologie de la poésie russe. La Renaissance du XX siècle, Paris, Aubier-Flammarion, 1970 (trad. par N. Struve), p. 47. 
C'est pourquoi la première tâche de la critique raisonnable d'une erreur est de déterminer la vérité qui lui sert de support et qu'elle déforme.

Le mauvais côté du nietzschéisme saute aux yeux. Mépris pour l'humanité faible et malade, point de vue païen sur la force et la beauté, fait de s'attribuer à l'avance une signification exceptionnelle surhumaine - premièrement à titre individuel, puis à titre collectif comme minorité d'élite des «meilleurs", c'est-à-dire des natures «de maitre», plus fortes, plus douées, enclines à exercer un pouvoir ${ }^{8}$, auxquelles tout est permis, en tant que leur volonté est la loi suprême pour les autres - voilà l'erreur évidente du nietzschéisme. En quoi consiste cette vérité, qui la rend forte et attrayante pour l'âme vivante?

La distinction entre vérité et erreur ne dispose pas même ici de deux mots séparés. C'est un seul et même mot qui réunit en lui et le mensonge et la vérité de cette étonnante doctrine. Toute la question est de savoir comment nous comprenons et prononçons le mot «surhomme». Dans ce mot est-ce la voix d'une prétention bornée et vide qui se fait entendre, ou bien la voix d'une conscience de soi profonde, ouverte aux meilleures possibilités et anticipant un avenir sans fin?

De toutes les créatures terrestres seul l'homme peut se rapporter à soimême de façon critique - non pas dans le sens d'une simple insatisfaction de telle ou telle de ses situations ou de ses actes (ce qui est possible également pour les autres animaux), ni non plus dans le sens d'un sentiment vague et indéterminé d'angoisse, propre à toute "créature gémissante " 9 , mais dans le sens d'une évaluation consciente négative du mode même de son être et des chemins fondamentaux de sa vie comme ne correspondant pas à ce qui devrait être. Nous nous jugeons nous-mêmes, et devant le tribunal de la raison nous nous accusons de bonne foi. Un certain gage d'une nature supérieure dans la profondeur de l'âme humaine nous contraint à vouloir un perfectionnement infini; la réflexion nous désigne le fait constant et universel de notre imperfection, et la conscience nous dit que ce fait n'est pas pour nous seulement une nécessité extérieure, mais dépend aussi de nous-mêmes.

Il est naturel à l'homme de vouloir être meilleur et plus grand que ce qu'il est en réalité, il lui est naturel de tendre à l'idéal du surhomme. S'il le veut vraiment, il le peut, et s'il le peut, il le doit. Mais n'est-ce pas là une absurdité - être meilleur, supérieur, davantage que sa réalité? Si, c'est une absurdité pour l'animal, en tant que pour lui la réalité est ce qui le fait et le gouverne; mais l'homme, quoiqu'il soit aussi l'œuvre d'une réalité déjà

8. «Властительный», de «власть»: «pouvoir», qui traduit l'allemand «Macht» dans la plupart des traductions russes de l'expression nietzschéenne "volonté de puissance».

9. Nouveau Testament, Romains 8, 22. 
donnée, qui existe avant lui, peut cependant agir sur elle de l'intérieur, et, en conséquence, sa réalité est d'une manière ou d'une autre, dans une certaine mesure, ce qu'il fait lui-même - qu'il fait de façon plus visible et évidente en qualité de créature collective, de façon moins visible, mais indubitablement tout autant en qualité de créature individuelle.

II. On peut discuter de la question métaphysique de la liberté inconditionnelle de choix, mais l'initiative de l'homme, sa capacité d'agir d'après des impulsions intérieures, des motifs plus ou moins dignes, et enfin d'après l'idéal même du bien parfait, n'est pas une question métaphysique, mais un fait de l'expérience psychique. Toute l'histoire ne parle d'ailleurs que de cela: comment l'homme collectif se fait meilleur et plus grand que lui-même, dépasse sa réalité présente ${ }^{10}$, la faisant reculer dans le passé et faisant s'avancer dans le présent ce qui encore récemment était contraire à la réalité - le rêve, l'idéal subjectif, l'utopie.

La croissance intérieure de l'homme et de l'humanité dans son principe réel est étroitement liée à ce processus de complexification et de perfectionnement de l'être naturel, à cette croissance cosmique qui s'exprime de façon particulièrement éclatante dans l'évolution des formes organiques de la vie végétale et animale. Avant l'apparition de l'homme, les formes de la vie sensible évoluent de façon large et variée; avec l'homme, l'évolution de la vie douée de raison commence de façon préhistorique et se poursuit sous les yeux de l'histoire. Du point de vue le plus objectif et réaliste - en laissant de côté toutes les distinctions discutables - il y a une distinction indiscutable, radicale et générale entre le monde de la nature et le monde de l'histoire, à savoir que la croissance de l'organisation physique se produit à travers l'élaboration progressive de nouvelles formes corporelles, qui à mesure que se poursuit le cours de l'évolution s'éloignent tellement des anciennes, leur deviennent si dissemblables, qu'on ne peut reconnaître tout de suite leur lien génétique. Qui par exemple, sans l'aide de la science, remarquerait la parenté naturelle du cheval et de l'escargot, du cerf et de l'huître, de l'alouette et de l'éponge, de l'aigle et du polype corallien, du palmier et du champignon?

C'est aussi ce changement d'aspect général et cette complexification des formes corporelles qui servent de support à l'évolution de la vie psychique des organismes (du moins dans le règne animal). Si la formation de nouvelles formes corporelles s'arrêtait, mettons, à la forme de l'huitre, il n'y aurait alors

10. «Наличная»: «disponible», «donnée», «effective», « existante». C’est ce qui est «en présence», «en face» : le mot dérive de «налицо» (de «на» [«devant»] et «лицо " [ le visage»]), mot utilisé pour parler de l'argent «en caisse», «comptant», «cash». 
non plus aucune évolution ultérieure en termes psychiques, car il est tout à fait évident que dans cette forme d'existence - l'huître - non seulement la création spirituelle de l'homme, mais même la vie psychique d'un chien, d'un singe ou ne serait-ce que d'une abeille ne pourraient trouver place. C'est donc qu'il fallait une longue série de nouvelles organisations corporelles comme autant de conditions de possibilité pour la croissance d'une vie intérieure, psychique. Mais voilà qu'avec l'apparition du corps humain, entre dans le monde une forme animale telle que grâce à l'évolution particulière en elle de l'appareil neuro-cérébral, elle n'exige plus de changements essentiels de son organisation corporelle, parce que cette même forme, tout en conservant tous ses traits typiques, tout en demeurant essentiellement identique, peut contenir en elle une série illimitée de degrés d'accroissement intérieur - psychique et spirituel - : depuis le sauvage à moitié animal, qui ne se sépare que presque seulement virtuellement du monde des autres animaux, jusqu'aux plus grands génies de la pensée et de la création.

Cette croissance intérieure, qui s'accomplit dans l'histoire, se reflète bien sûr aussi sur l'aspect extérieur de l'homme, mais dans des traits qui sont pour la biologie inessentiels et non typiques. La spiritualisation de l'apparence humaine ne modifie pas le type anatomique, et aussi haut que se hisse la contemplation du génie, la constitution de la tête du plus grossier des sauvages est pourtant identique à la sienne, et lui permet de regarder librement le ciel sans bornes.

III. L'histoire ne crée et n'exige aucune forme d'organisme nouvelle, surhumaine, car la forme humaine peut se perfectionner sans limites à la fois intérieurement et extérieurement, tout en demeurant identique: elle est capable de par son prototype, ou son type, d'inclure et de lier en elle tout ce à quoi l'on peut aspirer, d'en devenir l'outil et le porteur - elle est capable d'être la forme d'une unitotalité ${ }^{11}$ parfaite, ou d'une divinité.

Pareille stabilité morphologique et achèvement de l'homme comme type organique ne contredit en rien la vérité que nous reconnaissons de l'aspiration de l'homme à devenir plus grand et meilleur que sa réalité, ou à devenir surhomme, parce que la vérité de cette aspiration n'a pas trait à telles ou telles formes de l'être ${ }^{12}$ humain, mais seulement à son mode de

11. «Всеединство ». Concept capital dans l'œuvre de Soloviev qui désigne le fait que « tout est un», et apparaît dès sa thèse de doctorat: Critique des principes abstraits (1880): «J'appelle unitotalité vraie ou positive celle dans laquelle l'un (единое) n'existe pas sur le compte de tous ou à leurs dépens, mais à leur avantage. L'unité fausse, négative, écrase ou engloutit les éléments qui entrent en elle, sa vacuité apparait de cette façon d'elle-même; la vraie unité conserve et renforce ces éléments, en s'accomplissant en eux comme plénitude de l'être».

12. «Существо » désigne à la fois «l'essence», «la créature », «l'être». 
fonctionnement dans ces formes, qui n'est pas nécessairement lié à ces formes. Nous pouvons, par exemple, être insatisfaits de la condition réelle de la vue humaine, mais non pas bien sûr de ce que nous n'avons que deux yeux, seulement de ce que nous voyons mal avec eux. Car pour voir mieux, l'homme n'a aucun besoin de modification du type morphologique de son organe visuel. Il n'a aucunement besoin d'avoir une multitude d'yeux au lieu de deux, parce qu'avec ces deux yeux-là, la faiblesse de la vue (au sens littéral) devient modifiable par l'entremise des longues-vues, télescopes et microscopes inventés par l'homme lui-même; et en un sens plus élevé, avec ces deux yeux, peuvent s'ouvrir chez l'homme « des prunelles prophétiques, comme chez l'aigle épouvanté $»^{13}$, avec ces deux yeux-là, il peut devenir prophète et surhomme, alors qu'une créature ayant une autre forme organique, fût-elle dotée d'une centaine d'yeux, ne restera toujours qu'une mouche.

IV. De même, tout comme notre organe visuel, tout le reste de l'organisme humain dans aucun des traits normaux de sa constitution morphologique ne nous empêche de nous hisser au-dessus de notre basse réalité, et de devenir relativement à elle des surhommes. Les obstacles ici ne peuvent venir que du côté fonctionnel de notre existence et non seulement dans les déviations pathologiques rares et particulières, mais même dans ces phénomènes habituels que beaucoup sont enclins à cause de cela à considérer comme normaux.

Ainsi en est-il, avant tout et plus que tout, du phénomène de la mort. S'il est une chose qui nous pèse de façon bien naturelle, s'il est une chose dans la réalité présente dont nous sommes fondés à être insatisfaits, c'est bien, sans aucun doute, de ce phénomène final de toute notre existence visible, de ce terme évident, de cette réduction à rien. Un homme qui ne pense qu'à lui ne peut pas se réconcilier avec la pensée de $s a$ mort, un homme qui pense aux autres ne peut pas se réconcilier avec la pensée de la mort des autres: donc et l'égoïste, et l'altruiste - sachant qu'il est logiquement nécessaire pour tous les hommes d'appartenir à un degré divers de pureté ou de mélange à l'une ou à l'autre de ces catégories morales - tant l'égoïste que l'altruiste doivent pareillement ressentir la mort comme une contradiction intolérable, ils ne peuvent pareillement admettre comme définitif ce terme apparent de l'existence humaine. Voilà donc sur quoi, logiquement, les hommes désireux de se hisser au-dessus de la réalité donnée - désireux de devenir des surhommes - devraient concentrer leur

13. Citation tirée du poème de Pouchkine «Пророк» («Le Prophète»), v. 7-8, dans lequel le narrateur, touché par le doigt d'un Séraphin, voit tour à tour ses yeux, ses oreilles, sa langue, son cœur transfigurés ou remplacés par des organes nouveaux et surhumains qui élargissent sa perception. 
attention. Par quoi donc en effet se distingue en particulier cette humanité au-dessus de laquelle ils pensent s'élever, si ce n'est justement par ceci qu'elle est mortelle?

«Homme» et «mortel» sont synonymes. Chez Homère déjà, les hommes sont continuellement opposés aux dieux immortels justement comme

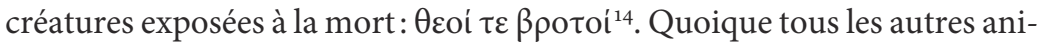
maux meurent, il ne vient à l'esprit de personne de les caractériser comme mortels - pour ce qui est de l'homme non seulement l'on admet cet attribut ${ }^{15}$ comme caractéristique, mais on sent encore dans le terme "mortel» une sorte de mélancolique reproche adressé à soi-même, on sent que l'homme, prenant conscience du caractère inéluctable de la mort comme particularité existentielle de sa condition réelle se refuse catégoriquement à se réconcilier avec elle, que la conscience de ce caractère inéluctable dans les conditions données ne l'apaise en rien. Et en cela, bien entendu, il est dans le vrai ${ }^{16}$, car si la mort est tout à fait nécessaire dans ces conditions présentes, qui a dit pour autant que ces conditions mêmes étaient immuables et inviolables?

L'animal ne lutte pas (consciemment) avec la mort et, en conséquence, ne peut être vaincu par elle, c'est pourquoi son caractère mortel ne prend en ce qui le concerne ni la forme d'un reproche ni une signification caractéristique; l'homme, lui, est au premier chef et de façon spécifique «mortel» - au sens de vaincu et battu par la mort. Mais s'il en est ainsi, alors le "surhomme» doit être au premier chef et de façon spécifique le vainqueur de la mort - le libéré qui libère l'humanité de ces conditions essentielles, qui rendent la mort nécessaire, et, en conséquence, celui qui remplit les conditions auxquelles il est possible ou bien de ne pas mourir du tout, ou bien, une fois mort, de ressusciter pour une vie éternelle. L'objectif ${ }^{17}$ est audacieux. Mais l'audacieux n'est pas seul, Dieu est avec lui, qui règne sur lui. Admettons que, même avec cette aide dans la condition actuelle de l'humanité, la victoire ne puisse être du tout atteinte dans les bornes de l'existence individuelle. Bien qu'il soit permis d'en douter, car il n'est pas possible de le prouver à l'avance, avant d'en faire l'expérience, mais admettons comme si cela était démontré que chacun d'entre nous, hommes du siècle qui s'achève et de celui qui advient, ainsi que des nombreux siècles à venir, mourra immanquablement, sans s'être préparé soi-même ni avoir préparé les autres à une résurrection immédiate. Mettons que le but est encore aussi éloigné qu'il

14. «Les dieux et les mortels» en grec ancien.

15. «Признак» est à la fois le «signe » et la traduction de la catégorie kantienne d' "attribut» par opposition à «substance».

16. «Он прав» est l'équivalent strict de «il a raison», mais l'adjectif «прав» s'apparente à vérité («правда») et non à raison («разум»).

17. «Задача » signifie à la fois «le problème», «la tâche», «l'objectif», «le but», «la mission ». 
s'est avéré éloigné pour ces chrétiens déraisonnables du premier siècle, qui pensaient que la vie éternelle dans des corps ressuscités et impérissables leur tomberait du ciel d'un moment à l'autre - mettons qu'elle soit éloignée aujourd'hui aussi. Mais le chemin qui y conduit, la façon dont nous nous en rapprochons par ce chemin, fût-ce lentement, dont se trouvent remplies, fût-ce imparfaitement, mais dans un mouvement continu de perfectionnement, les conditions dont la plénitude est requise pour le triomphe sur la mort - cela, c'est indubitablement possible, et cela existe réellement.

Ces conditions dans lesquelles la mort prend un empire sur nous, et nous vainc, elles nous sont assez connues, de par notre expérience tant personnelle que collective, de sorte que et par conséquent les conditions opposées ${ }^{18}$ devraient nous être également connues, dans lesquelles nous prenons un empire sur la mort et pouvons, en fin de compte, la vaincre.

V. Quand bien même ne se dresserait pas dans notre souvenir l'image d'un authentique "surhomme», d'un vainqueur réel de la mort, d'un "premier-né entre les morts ${ }^{19}$ (mais ne serait-ce pas là de notre part faire preuve d'une trop grande absence de mémoire?), ou quand bien même cette image serait si obscurcie et brouillée sous des strates diverses, qu'elle ne pût déjà plus rien dire à notre conscience de sa signification pour notre objectif vital (pourquoi cependant ne l'éclaircirions-nous et ne la débrouillerions-nous pas dans ce cas?) - quand bien même il n'y aurait pas devant nous de "surhomme" réel, de toute manière il y a le chemin surhumain, sur lequel beaucoup ont marché, marchent, et marcheront encore pour le bien de tous, et bien entendu, notre intérêt vital le plus important, c'est que davantage d'hommes s'engagent sur ce chemin, s'y avancent plus franchement et plus loin, car ce qui se trouve à sa fin, c'est la victoire pleine et décisive sur la mort.

Et voici le critère véritable d'évaluation de toutes les actions et de tous les phénomènes de ce monde: à quel point chacun d'entre eux correspond aux conditions nécessaires pour la régénération de l'homme mortel et souffrant dans un surhomme immortel et bienheureux. Et si la vieille forme

18. «Противоположный» («opposé») est l'adjectif formé sur «противоположность » qui signifie à la fois "contradiction" et "opposition». L'absence de distinction lexicale nette entre «contradictoire», «contraire» et «opposé» a des conséquences sur le sens, puisque plus haut le mot était employé dans un contexte apparenté, lorsqu'il s'agissait de faire advenir «ce qui encore récemment était contraire à la réalité - un rêve, un idéal subjectif, une utopie». Le texte fait bien appel à une forme singulière de «logique» qui serait calquée sur «l'expérience», comme l'indique le recours au vocabulaire de la "possibilité», de la «nécessité», des «conditions», de la «réalité» effective. Peut-être est-ce la marque d'un héritage hégélien.

19. Nouveau Testament, saint Paul, épître aux Colossiens 1, 18. 
traditionnelle de l'idée de surhomme s'est pétrifiée dans les esprits scolaires, a recouvert pour la multitude des hommes l'essence vivante de cette idée même, et a mené à son oubli - à l'oubli par l'homme de sa signification véritable et élevée -, à sa réconciliation avec le sort des autres créatures, ne convient-il pas de se réjouir déjà de ce simple fait que cet oubli et cette réconciliation pusillanime avec la réalité touche à sa fin, de ce que retentissent des voix qui déclarent, fût-ce encore de façon gratuite: "Je suis un surhomme", "Nous sommes des surhommes»? De telles déclarations, qui inspirent d'abord le dépit, doivent dans leur essence réjouir déjà en ceci qu'elles ouvrent la possibilité d'une discussion intéressante, ce qu'on ne peut pas dire de certains autres points de vue. À l'époque où je découpais des sangsues au rasoir et où je préférais le zoologue Haeckel au philosophe $\mathrm{Hegel}^{20}$, mon père m'a un jour raconté une anecdote assez connue sur la façon dont un négociant moscovite «arriéré» avait confondu un naturaliste «avancé», qui voulait le convertir au darwinisme. Cette doctrine, selon la mode d'alors, et "avec quelque malheur » pour Darwin lui-même, était comprise comme l'assimilation essentielle de l'homme aux autres animaux. Après avoir raconté beaucoup de choses sur ce sujet, le civilisateur ${ }^{21}$ " avancé» demande à l'auditeur: Tu as compris? - J'ai compris. - Eh bien qu'en dis-tu? - Qu'est-ce qu'on peut bien en dire? Si, donc, je suis un chien, et que toi, donc, tu es un chien, qu'est-ce donc qu'un chien peut avoir comme discussion avec un autre chien ${ }^{22}$ ?

Aujourd'hui, grâce à Nietzsche, les gens avancés se déclarent au contraire de telle façon qu'une discussion sérieuse est avec eux possible et requise - de plus sur des questions surhumaines. C'est à l'amorce ${ }^{23} \mathrm{~d}^{\prime}$ une telle discussion que j'ai voulu m'employer dans ces pages.

Vladimir Soloviev

Traduction et notes d'Antoine MULLER

20. Soloviev joue sur la sonorité voisine des deux noms, qui symbolisent deux grandes influences successives qui se sont exercées à tour de rôle en Russie (voir par exemple I. Tourgueniev, Pères et fils, paru en 1862), d'autant plus que la transcription russe (Гегель / Геккель) renforce encore la ressemblance.

21. «Просветитель» est le substantif formé sur «Просвещение»: «les Lumières».

22. Il n'est pas impossible qu'il y ait là une réminiscence des conversations des petits chiens Medji et Fidèle dans le Journal d'un fou de Gogol (3 octobre, 12 et 13 novembre).

23. «Приступ» («assaut») est d'abord un terme guerrier, qui signifie «attaquer» au sens d' «entamer» ou "aborder», ou l'«accès " par lequel se déclare une maladie. Soloviev est mort quelques mois plus tard sans avoir pu mener cette discussion à bien. 